PHYSICAL REVIEW D 96, 019902(E) (2017)

\title{
Erratum: Discrimination of dark matter models in future experiments [Phys. Rev. D 91, 095004 (2015)]
}

Tomohiro Abe, Ryuichiro Kitano, and Ryosuke Sato

(Received 23 June 2017; published 12 July 2017)

DOI: $10.1103 /$ PhysRevD.96.019902

There is a typographical error in the original paper. The integral form of $H\left(m_{1}, m_{2}\right)$ in Appendix A [Eq. (A4)] should be

$$
H\left(m_{1}, m_{2}\right)=\frac{1}{\hat{\epsilon}}\left(\frac{2}{3} p^{2}-m_{1}^{2}-m_{2}^{2}\right)-\int_{0}^{1} d x\left(4 x(1-x) p^{2}-2 x m_{1}^{2}-2(1-x) m_{2}^{2}\right) \log \frac{\Delta}{\mu^{2}},
$$

where $\Delta=x m_{1}^{2}+(1-x) m_{2}^{2}-x(1-x) p^{2}-i \epsilon$. 